scendental and an algebraic function. Thus we reached a contradiction and $P(x) \equiv 0$ which means that $\int_{0}^{1}(P(x))^{2} d x=0$.

\title{
REFERENCE
}

1. B. Nyman, On the one-dimensional translation group and semi-group in certain function spaces, Dissertation, Univ. of Uppsala, 1950. Math. Rev. 12 (1951), 108; Zbl. Math. 37 (1951), 354.

Wayne State University

\section{A NOTE ON THE LIPSCHITZ CONDITION IN METRIC SPACES}

NORMAN LEVINE

Let $f: M_{1} \rightarrow M_{2}$ be a single valued transformation where $M_{1}$ and $M_{2}$ are metric spaces with metrics $d_{1}$ and $d_{2}$ respectively.

Definition. $f: M_{1} \rightarrow M_{2}$ is termed Lipschitzian if and only if there exists a finite constant $K$ such that $d_{2}(f(a), f(b)) \leqq K d_{1}(a, b)$ for all $a, b \in M_{1}$.

THEOREM. Let $\left\{M_{i}, d_{i}\right\}$ be an infinite sequence of metric spaces and $f_{i, j, k}: M_{i} \rightarrow M_{j}$ continuous for all positive integers $i, j$ and $k$. Then for each $i$ there exists a metric $d_{i}^{*}$ for $M_{i}$ which is equivalent to $d_{i}$ such that $f_{i, j, k}: M_{i} \rightarrow M_{j}$ is Lipschitzian relative to $d_{i}^{*}$ and $d_{j}^{*}$ for all $i, j$ and $k$.

Proof. Without loss of generality we may assume that each $d_{i}$ is bounded by 1 on $M_{i}$. Let $a, b \in M_{\text {s }}$ and define

$$
\begin{aligned}
d_{s}^{*}(a, b) \equiv d_{s}(a, b)+\sum_{q=1}^{\infty} 1 / 2^{q+n_{1}+\cdots+n_{q}+k_{1}+\cdots+k_{q}} \\
\cdot d_{n_{1}}\left(f_{n_{2}, n_{1}, k_{1}} \cdots f_{n_{q}, n_{q-1}, k_{q-1}} f_{s, n_{q}, k_{q}}(a), \cdots(b)\right),
\end{aligned}
$$

where the summation runs over all $n_{1}, \cdots, n_{q} \in S_{q}$ and $k_{1}, \cdots, k_{q}$ $\in S_{q}, S_{q}$ being the set of all finite sequences of positive integers with $q$ elements; juxtaposition of the $f$ 's of course means composition. Then $d_{s}^{*}$ satisfies all of the axioms for a metric on $M_{s}$. The proof will be complete when we show that (1) $d_{s}^{*}$ is equivalent to $d_{s}$ on $M_{s}$ and (2) $f_{i, j, k}: M_{i} \rightarrow M_{j}$ is Lipschitzian relative to $d_{i}^{*}$ and $d_{j}^{*}$. To show (1) let $y \in M_{0}$, and $\left\{y_{n}\right\}$ be an infinite sequence in $M_{s}$. Suppose $\lim _{n} d_{s}^{*}\left(y, y_{n}\right)=0$. Then $\lim _{n} d_{s}\left(y, y_{n}\right)=0$ since $d_{s}\left(y, y_{n}\right) \leqq d_{s}^{*}\left(y, y_{n}\right)$. Conversely suppose $\lim _{n} d_{s}\left(y, y_{n}\right)=0$. We note first that

Received by the editors December 29, 1960, and, in revised form, March 18, 1961. 
$d_{s}^{*}(a, b) \leqq 1+\sum_{q=1}^{\infty} 1 / 2^{q+n_{1}+\cdots+n_{q}+k_{1}+\cdots+k_{q}} \quad$ (summed over $S_{q}$ as above)

for all $a, b$ in $M_{s}$. Hence the series representation for $d_{s}^{*}(a, b)$ is uniformly convergent, and each term is a continuous function of the arguments $a$ and $b$. Thus $\lim _{n} d_{s}^{*}\left(y, y_{n}\right)=d_{s}^{*}\left(y, \lim _{n} y_{n}\right)=d_{s}^{*}(y, y)=0$. To show (2) consider $f_{i, j, k}: M_{i} \rightarrow M_{j}$. Take $a$ and $b$ in $M_{i}$. Then

$$
\begin{aligned}
d_{j}^{*}\left(f_{i, j, k}(a), f_{i, j, k}(b)\right)= & d_{j}\left(f_{i, j, k}(a), f_{i, j, k}(b)\right) \\
& +\sum_{\substack{q=1 \\
n_{1}, \cdots, n_{q} \in S_{q} \\
k_{1}, \cdots, k_{q} \in S_{q}}}^{\infty} 1 / 2^{q+n_{1}+\cdots+n_{q}+k_{1}+\cdots+k_{q}} \\
& d_{n_{1}}\left(f_{n_{\mathbf{q}}, n_{1}, k_{1}} \cdots f_{j, n_{q}, k_{q}} f_{i, j, k}(a), \cdots(b)\right) \\
= & 2^{1+j+k}\left\{1 / 2^{1+j+k} d_{j}\left(f_{i, j, k}(a), f_{i, j, k}(b)\right)\right. \\
& +\sum_{\substack{n_{1}=1 \\
k_{1}, \cdots, n_{q} \in S_{q}}}^{\infty} 1 / 2^{q+1+n_{1}+\cdots+n_{q}+j+k_{1}+\cdots+k_{q}+k} \\
& \left.\cdot d_{n_{1}}\left(f_{n_{2}, n_{1}, k_{1}} \cdots f_{j, n_{q}, k_{q}} f_{i, j, k}(a), \cdots(b)\right)\right\} \\
\leqq & 2^{1+j+k} d_{i}^{*}(a, b) .
\end{aligned}
$$

Thus $d_{j}^{*}\left(f_{i, j, k}(a), f_{i, j, k}(b)\right) \leqq K d_{i}^{*}(a, b)$ where $K=2^{1+j+k}$ and the proof is complete.

That the theorem has no generalization to the uncountable case is evident from the following example:

Let $M_{1}$ be the space of rationals and $M_{2}$ be the interval $[0,1]$, both with the usual topology. Let $d_{1}$ and $d_{2}$ be metrics for $M_{1}$ and $M_{2}$ respectively (not necessarily the natural metrics). Now $\left\{M_{1}, d_{1}\right\}$ is not complete since any complete, dense-in-itself space is uncountable. Suppose further that $\left\{f_{\alpha}\right\}$ is the class of all continuous mappings from $M_{1}$ to $M_{2}$. We assert that at least one of the $f_{\alpha}$ is not uniformly continuous (and hence not Lipschitzian) relative to $d_{1}$ and $d_{2}$. Since $\left\{M_{1}, d_{1}\right\}$ is not complete we may take $\left\{x_{k}\right\}$, a Cauchy sequence of distinct points which is not convergent. Let $F \equiv \bigcup\left\{x_{2 n}\right\}$ and $G \equiv \bigcup\left\{x_{2 n-1}\right\} . \quad F$ and $G$ are nonempty, closed, and disjoint. By Urysohn's Lemma there exists a continuous map $g: M_{1} \rightarrow M_{2}$ such that $g(F)=0$ and $g(G)=1$. Then $g \in\left\{f_{\alpha}\right\}$. But $g: M_{1} \rightarrow M_{2}$ is not uniformly continuous since $d_{1}\left(x_{2 n}, x_{2 n-1}\right)$ converges to 0 , but $d_{2}\left(g\left(x_{2 n}\right), g\left(x_{2 n-1}\right)\right)$ does not converge to 0 .

Ohio State University 